\title{
Vehicular ADHOC Network (VANET):-A Brief Knowledge
}

\author{
Sandeep N. Kugali ${ }^{1}$ \\ ${ }^{1}$ Department of Information Science and Engineering, \\ Basaveshwar Engineering College (Autonomous), \\ Bagalkot, Karnataka, INDIA
}

\author{
Sneha Kadadevar ${ }^{2}$ \\ ${ }^{2}$ Department of Computer Science, Basaveshwar Arts \\ Science and Commerce Independent PU College, Bagalkot, \\ Karnataka, INDIA
}

\begin{abstract}
Vehicular ad hoc network is one of the most interesting research areas due to flexibility, low cost, high sensing fidelity, fault tolerance, creating many new and exciting application areas for remote sensing. So, it has emerged as a promising tool for monitoring the physical world with wireless sensor that can sense, process and communicate. Being ad-hoc in nature, VANET is a type of networks that is created from the concept of establishing a network of cars for a specific need or situation. VANETs have now been established as reliable networks that vehicles use for communication purpose on highways or urban environments. VANET considered as a distinct type of Mobile Ad Hoc Networks holds the opportunity to make people's life and death decisions by predicting and helping the drivers and other people about the road safety and other critical conditions. This paper outlines the VANET definition, its architecture and protocols from a research point of view and future research directions of VANET are provided in the end of paper.
\end{abstract}

Keywords: Ad-hoc networks, Vehicles, Routing, Security

\section{INTRODUCTION}

VANET refers to a network created in an ad-hoc manner where different moving vehicles and other connecting devices come in contact over a wireless medium and exchange useful information to one another. A small network is created at the same moment with the vehicles and other devices behaving as nodes in the network. Whatever information the nodes possess is transferred to all other nodes. Similarly all the nodes after transferring their set of data receive the data being transmitted by other nodes. After accumulating all of such data, nodes then work to generate useful information out of the data and then again transmit the information to other devices [1][2]. The communication between devices expands in such as way that the where nodes are free to join and leave the network i.e. it is an open network. The new vehicles being launched in the market are now coming with equipped on board sensors which make it easy for the vehicle to easily join and merge in the network and leverage the benefits of VANET.

Vehicular Ad-hoc Networks are expected to implement a variety of wireless technologies such as Dedicated Short Range Communications (DSRC) which is a type of Wi-Fi. Other Wireless Technologies are Cellular, Satellite and WiMAX. Vehicular Ad-hoc Networks can be viewed as component of the [3] Intelligent Transportation Systems (ITS). Vehicular ad-hoc networks are responsible for the communication between moving vehicles in a certain environment. A vehicle can communicate with another vehicle directly which is called Vehicle to Vehicle (V2V) communication, or a vehicle can communicate to an infrastructure such as a Road Side Unit (RSU), known as Vehicle-to-Infrastructure (V2I).

VANET can be characterized by the following factors [4], [5]:

- Network topology: Due to high node mobility and random speed of vehicles, the position of node changes frequently. As a result of this, network topology in VANETs tends to change frequently.

- Unbounded network size: VANET can be implemented for one city, several cities or for countries. This means that network size in VANET is geographically unbounded.

- Frequent exchange of information: The ad hoc nature of VANET motivates the nodes to gather information from the other vehicles and road side units.

- Unlimited power and storage: It is assumed that the nodes in VANET are capable of possessing an unlimited amount of power as well as storage capacity. Therefore the nodes are free to exchange the data without the foundations of power consumption or storage wastage.

- On board sensors: VANET assumes that the nodes are seldom equipped with on board sensors which are capable of transmission of information to other devices or nodes.

The rest of the paper is organized as: II. Explains the architecture for a VANET. III. Explains the transmission or broadcasting protocols being used by VANET for communication among various nodes. IV. Explains the real world applications of VANET. V. Outlines the future research challenges for VANET and VI. Concludes the paper.

\section{ARCHITECTURE}

According to IEEE 1417-2000 and ISO/IEC 42010 architecture and guideline VANET system can be divided into three domains. The mobile domain, the infrastructure domain, and the generic domain Fig.1.

- Mobile domain: Mobile domain comprises of two parts. First is vehicle domain which encompasses all the vehicles which are moving constantly such as buses, cars, trucks etc. Second part is mobile device domain which comprises of all the portable handy devices such as PDAs, laptop, GPS, smart phones etc. 
- Infrastructure domain: It also comprises of two parts. Roadside infrastructure domain comprises of stationary roadside entities such as traffic lights, poles etc. Whereas, central infrastructure domain encompasses the central managing centre such as vehicle management centre, traffic management centre etc.

- Generic domain: It comprises of Internet infrastructure and Private infrastructure. For instance, different nodes and servers and other computing resources working directly or indirectly for a VANET come under generic domain.

The mobile domain exchanges information and communicates to Infrastructure domain which processes data and does its own modulation. Then in the second step, infrastructure domain in turn communicates to generic domain and exchanges information with it. This data flow among the stationary and mobile resources results in efficient and effective utilization of road by the users [5]. Another form of VANET architecture is communication architecture where communication types are characterized into 3 sections which are briefed as [6] Fig. 2:

- Vehicle to Vehicle Communication: It refers to inter vehicle communication. Vehicles or a group of vehicles connect with one another and communicate like point to point architecture. It proves to be very helpful for cooperative driving.

- Vehicle to Infrastructure Communication: Number of base stations positioned in close proximity with a fixed infrastructure to the highways is necessary to provide the facility of uploading/downloading of data from/to the vehicles. Each infrastructure access point covers a cluster.

- Cluster to Cluster Communication: In VANETs network is split into clusters that are self managed group of vehicles. Base Station Manager Agent (BSMA) enables communications between the clusters. BSMA of one cluster communicates with that of other cluster.

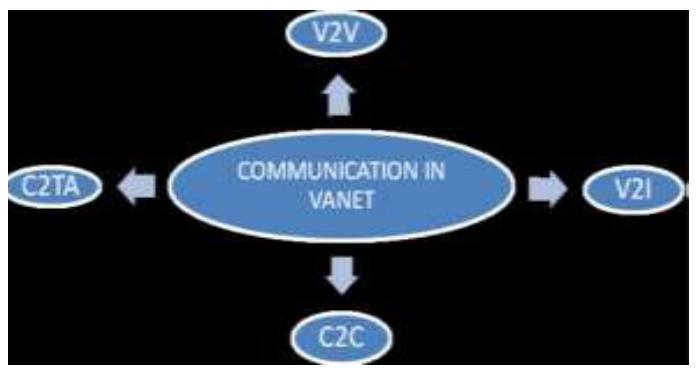

Fig.2: VANET Communication Types

\section{PROTOCOLS FOR TRANSMISSION}

The data being gathered and exchanged by the vehicles requires some protocols or rules through which transmission can take place in a systematic and organized way. The data exchange between nodes in a VANET happens via

Routing protocols. These protocols define how a packet of data will be distributed among different nodes. In VANET, the routing protocols are classified into five categories: Topology based routing protocol, Position based routing protocol, Cluster based routing protocol, Geo cast routing protocol and Broadcast routing protocol. These protocols are characterized on the basis of area / application where they are most suitable [7].

- Topology Based Routing Protocols: These routing protocols use links information that exists in the network to perform packet forwarding. They are further divided into Proactive and Reactive. Proactive means that the routing information, like next forwarding hop is maintained in the background irrespective of communication requests. The various types of proactive routing protocols are: LSR, FSR. Reactive routing opens the route only when it is necessary for a $\mathrm{n}$ ode to communicate with each other. The various types of reactive routing protocols are $\mathrm{AODV}, \mathrm{PGB}, \mathrm{DSR}$ and TORA

- Position Based Routing Protocols: Position based routing consists of class of routing algorithm. They share the property of using geographic positioning information in order to select the next forwarding hops. The packet is send without any map knowledge to the one hop neighbor, which is closest to destination. Position based routing is broadly divided in two types: Position based greedy V2V protocols, Delay Tolerant Protocols. Position Based Greedy V2V Protocols In greedy strategy and intermediate node in the route forward message to the farthest neighbor in the direction of the next destination. Greedy approach requires that intermediate node should possessed position of itself, position of its neighbor and destination position e.g., GPCR, CAR, DIR. The Delay Tolerant Protocols In urban scenario where vehicle are densely packed locating a node to carry a message is not a problem but in rural highway situation or in cities at night fewer vehicles are running and establishing end to end route is difficult. So in such cases certain consideration needs to be given in sparse networks. The various types of Delay Tolerant Protocols are MOVE, VADD, and SADV. 
- Cluster Based Routing Protocols: Cluster based routing is preferred in clusters. A group of nodes identifies themselves to be a part of cluster and a $\mathrm{n}$ ode is designated as cluster head will broadcast the packet to cluster. $\mathrm{T}$ he various Clusters based routing protocols are COIN and LORA_CBF.

- Geo-cast Routing Protocols: Geo cast routing is basically a location based multicast routing. Its objective is to deliver the packet from source node to all other nodes within a specified geographical region (Zone of Relevance ZOR). In Geo cast routing vehicles outside the ZOR are not alerted to avoid unnecessary hasty reaction. Geo cast is considered as a multicast service within a specific geographic region. The various Geo cast routing protocols are IVG, DG-CASTOR and DRG.

- Broadcast routing protocols: Broadcast routing is frequently used in VANET for sharing, traffic, weather and emergency, road conditions among vehicles and delivering advertisements and announcements. The various Broadcast routing protocols are BROADCOMM, UMB, VTRADE, and DV-CAST.

\section{IV. APPLICATIONS}

The four major classes of applications possible in VANET are safety oriented, convenience oriented and commercial oriented [8], [9]. Safety applications will monitor the surrounding road, approaching vehicles, surface and curves of the road. Convenience applications will be mainly of traffic management type. Commercial applications will provide the driver with the entertainment and services as web access, streaming audio and video. Productive applications are additional to the above mentioned applications.

- Safety Applications: This is again classified into RealTime traffic, Co-operative Message Transfer, Post Crash Notification, Road Hazard Control Notification, Cooperative Collision Warning, and Traffic Vigilance.

- Convenience Applications: This is again classified into Route Diversions, Electronic Toll Collection, Parking Availability, and Active Prediction.

- Commercial Applications: These are classified into Remote Vehicle Personalization/ Diagnostics, Internet Access, Digital map downloading, Real Time Video Relay, Value-added advertisement

- Productive Applications: These are classified into Environmental Benefits, Time Utilization, and Fuel Saving.

\section{CHALLENGES AND FUTURE RESEARCH DIRECTIONS}

The challenges can be viewed as a future research direction or open research issues where advancement and solutions are still required. Some of these challenges which user can take as research issues are [10], [11]:

- Vehicular Security Challenges: VANET face many security attacks and these attacks and threat can be categorized in few classes. The five different types of classes for the attacks with the aim to provide an easy identification with association to respective class. Each of the classes will represent different types of attack level and priority. The proposed classes of attack are Network Attack, Denial of Service attack, Sybil Attack, Application Attack, Fabrication Attack, Alteration Attack, Social Attack, Tunnel Attack, Monitoring Attack, Eavesdropping.

- Vehicular Networks Challenges: Mobility, Volatility, Privacy VS Authentication , Privacy VS Liability, Network Scalability, Bootstrap

- Vehicular Technical Challenges: Network Management, Congestion and collision Control, Environmental Impact, MAC Design, Security

- QoS: QoS support over VANETs remains a challenge when current routing paths become no longer available as a result of changes in node velocity, node positioning, network topology or distance between vehicular nodes. It may be a challenging issue both for network engineers and researchers to utilize the available bandwidth allocated for VANET to improve delivery of messages as well as to develop adaptive QoS routing protocols that will establish new routes quickly and efficiently.

- Routing: In order to timely and properly sending data packets from one node to another node an efficient routing algorithm is required. In VANET, efficient routing algorithm means a routing scheme with minimum delay, maximum system capacity and less computational complexity. Design such an algorithm which can be implemented in multiple topologies of the network and satisfies all of the above mentioned properties is an active area of research in VANET.

\section{CONCLUSION}

VANET is a promising wireless communication technology for improving highway safety and information services. Still, there are several challenges ahead, the profitable impact of VANETs on traffic safety and efficiency must be shown. This paper outlines the basic overview of what a VANET is all about. Further sections elaborate the architecture of VANET from system and communication point of view followed by the types of protocols being used by VANET. Application areas of VANET are explained in the next section from the research point of view. Finally, open research challenges which still need addressing are presented followed by conclusion of the paper.

\section{REFERENCES}

[1] Boto Bako and MichaelWeber, "Efficient Information Dissemination in VANETs Institute of Media Informatics, Ulm University Germany.

[2] Rakesh Kumar and Mayank Dave,"A Review of Various VANET Data Dissemination Protocols", International Journal of u- and e- Service, Science and Technology Vol. 5, No. 3, September, 2012.

[3] Amit Kumar Saha and David B. Johnson,"Modeling Mobility for Vehicular Ad Hoc Networks", VANET'04, ACM 1-58113-9225/04/0010, Philadelphia, Pennsylvania, USA, October 1, 2004.

[4] Manjyot Saini, Harjit Singh," VANET, its Characteristics, Attacks and Routing Techniques: A Survey, International Journal of Science and Research (IJSR), Volume 5 Issue 5, May 2016. 
[5] Ravi Tomar, Manish Prateek, G. H. Sastry," Vehicular Adhoc Network (VANET) - An Introduction, pp. 8883-8888, I J C T A, 9(18) 2016.

[6] Dr. Pankaj Dadhich, Priyanka Vyas, "Vehicular Ad Hoc Network (VANETs): A Brief Overview", Journal of Advanced Computing and Communication Technologies (ISSN: 2347 - 2804), Volume No.5 Issue No.3, June 2017.

[7] Rakesh Kumar and Mayank Dave, "A Comparative Study of Various Routing Protocols in VANET", IJCSI International Journal of Computer Science Issues, Vol. 8, Issue 4, No 1, July 2011.

[8] Kamini and Rakesh Kumar, "VANET Parameters and Applications: A Review", Global Journal of Computer Science and Technology, Vol. 10 Issue 7 Ver. 1.0 September 2010.
[9] Vishal Kumar, Shailendra Mishra and Narottam Chand, “ Applications of VANETs: Present \& Future", Communications and Network, 2013, 5, 12-15 doi:10.4236/cn.2013.51B004 Published Online February 2013.

[10] Ms. Divyalakshmi Dinesh and Prof. Manjusha Deshmukh, "Challenges in Vehicle Ad Hoc Network (VANET)", International Journal of Engineering Technology, Management and Applied Sciences, Volume 2 Issue 7, ISSN 2349-4476, December 2014.

[11] Er.Gurpreet Singh, " Overview of Challenges in VANET", International Journal of Innovative Research in Science and Engineering, Vol.No.2, Issue 08, August 2016. 